\title{
ON THE RATE OF CONVERGENCE OF SOME STOCHASTIC PROCESSES*†
}

\author{
WALTER KERN \\ Universität zu Köln
}

\begin{abstract}
We present a general technique for obtaining bounds on the deviation of the optimal value of some stochastic combinatorial problems from their mean. As a particular application, we prove an exponential rate of convergence for the length of a shortest path through $n$ random points in the unit square. This strengthens a previous result of Steele [St (1981b)].
\end{abstract}

1. Introduction. Let $X_{1}, X_{2}, \ldots$ be i.i.d. random variables taking values in $U=[0,1]^{d}$. For each $n$, let $Y_{n}$ denote a real-valued random variable which is measurable with respect to the $\sigma$-algebra generated by $X_{1}, \ldots, X_{n}$. Then there exists a Borel measurable function $f_{n}: U^{n} \rightarrow \mathbb{R}$ such that $Y_{n}=f_{n}\left(X_{1}, \ldots, X_{n}\right)$. Then, under certain assumptions concerning $f_{n}$ and, maybe, the distribution of the $X_{i}$ 's, one can prove limit theorems of the following type:

$$
\lim _{n \rightarrow \infty} \frac{1}{\delta(n)}\left|Y_{n}-E Y_{n}\right|=0 \quad \text { a.e. }
$$

with $\delta(n)=n$ in case $d=1$ (cf. [Ki (76)]) and $\delta(n)=n^{(d-1) / d}$ if $d \geqslant 2$ (cf. [St (81a)]). In particular such convergence results exist in case $Y_{n}$ denotes the optimal value of a combinatorial optimization problem defined by $X_{1}, \ldots, X_{n}$. Let us look at some examples.

EXAMPLE 1 (cf. [Rh (86)]). Let $d=1$, i.e. $\left(X_{i}\right)$ is a sequence of i.i.d. random variables taking on values in $[0,1]$, and let $Y_{n}$ denote the minimum number of bins to pack $n$ items of size $X_{1}, \ldots, X_{n}$. Then (*) holds without any further assumption on the distribution of the $X_{i}^{\prime}$ 's.

EXAMPLE 2 (cf. [BHH (59)]). Let $d=2$ and let $Y_{n}$ denote the shortest path connecting $X_{1}, \ldots, X_{n}$. Then $(*)$ holds, provided the $X_{i}$ 's are uniformly distributed in $[0,1]^{2}$ (cf. [St (81a)] for nonuniform distributions).

Further examples may be derived from the Subset Sum Problem, the Steiner Tree Problem and many others.

In [St (81a)] limit theorems have been proven without restricting the random variables to $[0,1]^{d}$ (however some kind of bounded support is still essentially used). From a practical point of view the assumption that the $X_{i}$ 's are bounded is much less restrictive than the assumptions on the distribution of the $X_{i}$ 's, which are sometimes used to prove convergence.

\footnotetext{
*Received February 26, 1987; revised December 8, 1987.

AMS 1980 subject classification. Primary: 60F05, 68C25. Secondary: 05B40.

IAOR 1973 subject classification. Main: Probability. Cross references: Graphs.

OR/MS Index 1978 subject classification. Primary: 561 Probability. Secondary: 488

Networks/Graphs/Stochastic.

Key words. Stochastic combinatorics, traveling salesman problem, bin-packing, convergence rates

${ }^{\dagger}$ Supported by the German Research Association (Deutsche Forshungsgemeinschaft, SFB 303).
} 
A further subject, which may be of interest in practice, is the rate of convergence in (*). There are two results which have been obtained in special cases (cf. Examples 1 and 2):

Proposition 1.1 (cf. [Rh (86)]). Let $\left(X_{i}\right)$ be a sequence of i.i.d. random variables táking on values in $[0,1]$ and let $Y_{n}$ denote the minimum number of bins to pack items of sizes $X_{1}, \ldots, X_{n}$. Then there exists a constant $C>0$ (independent of the distribution of the $X_{i}^{\prime}$ 's) such that

$$
\operatorname{Prob}\left(\frac{1}{n}\left|Y_{n}-E Y_{n}\right|>t\right) \leqslant C e^{-t \sqrt{n}}
$$

Proposition 1.2 (cf. [St $(81 \mathrm{~b})]$ ). Let $X_{i}$ be a sequence of i.i.d. random variables taking on values in $[0,1]^{2}$ and let $Y_{n}$ denote the length of a shortest path through $X_{1}, \ldots, X_{n}$. If the $X_{i}$ 's are uniformly distributed, then for every $k \in \mathbb{N}$ there exists $a$ constant $c_{k}>0$ such that

$$
\operatorname{Prob}\left(\frac{1}{n}\left|Y_{n}-E Y_{n}\right|>t\right) \leqslant c_{k}(t \sqrt{n})^{-k}
$$

Of course, this result is weak, compared with the exponential rate of convergence in Proposition 1.1. In $\$ 2$ we will see that in fact the two-dimensional case is the one which is difficult. It will follow that under very weak conditions, the rate of convergence is exponential whenever $d \neq 2$. For the two-dimensional case, somewhat stronger conditions will imply an exponential rate. These stronger conditions, however, seem to be satisfied by most of the "combinatorial optimization" functions $f$ (e.g. they are valid in case $f$ denotes the length of a shortest path).

2. The main theorem. Let $X_{1}, X_{2}, \ldots$ be i.i.d. random variables taking on values in $\mathbb{R}^{d}$. For $n \geqslant 2$, let $Y_{n}$ be a real-valued random variable which is measurable with respect to the $\sigma$-algebra generated by $X_{1}, \ldots, X_{n}$. Let $f: \mathbb{R}^{\text {nd }} \rightarrow \mathbb{R}$ be a Borel measurable function such that $Y_{n}=f\left(X_{1}, \ldots, X_{n}\right)$. Define "missing one"-functions $f_{i}^{n}: \mathbb{R}^{(n-1) d} \rightarrow \mathbb{R}$ by

$$
f_{i}^{n}\left(x_{1}, \ldots, x_{n}\right):=f_{n-1}\left(x_{1}, \ldots, x_{i-1}, x_{i+1}, \ldots, x_{n}\right) .
$$

Suppose that there exists constant $A>0$, such that

$$
\left|f_{i}^{n}-f_{j}^{n}\right| \leqslant A \quad \forall i, j \quad \forall n
$$

Furthermore, suppose that there exist a constant $B>0$ and $\gamma: \mathbb{N} \rightarrow \mathbb{R}_{+}$such that

$$
\sum_{i=1}^{n} \sum_{j=1}^{n}\left|f_{i}^{n}-f_{j}^{n}\right|^{2} \leqslant B \gamma(n) \quad \forall n .
$$

For reasons to become clear later, we assume that $\gamma(n) \geqslant n$. Then the following holds:

THEOREM 2.1. There exist constants $\alpha, \beta>0$, depending only on $A$ and $B$, such that for all $n$

$$
\operatorname{Prob}\left(\left|Y_{n}-E Y_{n}\right|>t\right) \leqslant \beta e^{-\alpha t \sqrt{n / \gamma(n)}}
$$


Before giving the proof, let us consider some examples. In the Bin Packing Problem (Example 1 of the preceding section), we may choose $A=1, B=1, \gamma(n)=n^{2}$. This yields for $t>0$ :

$$
\operatorname{Prob}\left(\left|Y_{n}-E Y_{n}\right|>t\right) \leqslant \beta e^{-\alpha t / \sqrt{n}} \text { or } \operatorname{Prob}\left(\frac{1}{n}\left|Y_{n}-E Y_{n}\right|>t\right) \leqslant \beta e^{-\alpha t \sqrt{n}}
$$

which is essentially Proposition 1.1.

Note that in general, whenever the first condition $\left|f_{i}^{n}-f_{j}^{n}\right| \leqslant A$ is satisfied (which usually follows from restricting the $X_{i}$ 's to $[0,1]^{d}$ ), then the second condition always is satisfied with $B=A^{2}$ and $\gamma(n)=n^{2}$. From this it is immediate that the rate of convergence in (*) (cf. §1) is exponential for $d \neq 2$. In case $d=2$, however, $\gamma(n)<n^{2}$ is needed for an exponential rate of convergence and this may sometimes be a little more difficult to prove. However, I feel that in most cases, when the $X_{i}$ 's are restricted to $[0,1]^{d}$ and $Y_{n}$ denotes the optimal value of some combinatorial optimization problem, then an expotential rate of convergence can be proved even in case $d=2$. Let us consider the Shortest Path Problem (cf. Example 2 of $\S 1$ ). Thus let $X_{i} \in[0,1]^{2}$ and let $f_{n}$ denote the length of a shortest path. Then the first condition is trivially satisfied with $A=2 \sqrt{2}$. The following lemma shows that the second condition can be fulfilled with $\gamma(n)=n \log n$.

LEMMA 2.2. Let $x_{1}, \ldots, x_{n}$ be the points in the unit square $[0,1]^{2}$ and let $f_{i}^{n}$ denote the length of a shortest path through $x_{1}, \ldots, x_{i-1}, x_{i+1}, \ldots, x_{n}$. Then there exists $B>0$ such that

$$
\sum_{i=1}^{n} \sum_{j=1}^{n}\left|f_{i}^{n}-f_{j}^{n}\right|^{2} \leqslant B n \log n
$$

Proof. A simple application of the triangle inequality shows that

$$
\left|f_{i}^{n}-f_{j}^{n}\right| \leqslant 2 \max \left(d_{i}, d_{j}\right)
$$

where $d_{i}$ is the minimal distance between $x_{i}$ and any of the other points. Now we have $\left|f_{i}^{n}-f_{j}^{n}\right|^{2} \leqslant 2^{2} \max \left(d_{i}, d_{j}\right)^{2} \leqslant 4\left(d_{i}^{2}+d_{j}^{2}\right)$. Thus

$$
\sum_{i, j}\left|f_{i}^{n}-f_{j}^{n}\right|^{2} \leqslant 4 \sum_{i, j}\left(d_{i}^{2}+d_{j}^{2}\right) \leqslant 4 n \sum_{i} d_{i}^{2}
$$

But

$$
\sum_{i} d_{i}^{2} \leqslant c^{2}\left(\frac{1}{n}+\frac{1}{n-1}+\cdots+\frac{1}{2}\right)
$$

using the fact that any $m$ points in $[0,1]^{2}$ have a pair at distance at most $c / \sqrt{m}$ and the obvious sequential argument.

Hence we may apply Theorem 2.1 with $\gamma(n)=n \log n$ which yields

$$
\operatorname{Prob}\left(\left|Y_{n}-E Y_{n}\right|>t\right) \leqslant \beta e^{-\alpha t / \sqrt{\log n}} \text { or } \operatorname{Prob}\left(\frac{\left|Y_{n}-E Y_{n}\right|}{\sqrt{n}}>t\right) \leqslant \beta e^{-\alpha t \sqrt{n / \log n}} .
$$

Note that in case $\gamma(n)<n^{2}$, Theorem 2.1 implies convergence a.e. in the sense that

$$
\sum_{n \geqslant 0} \operatorname{Prob}\left(\frac{1}{\sqrt{n}}\left|Y_{n}-E Y_{n}\right|>t\right)<\infty \quad \forall t>0
$$


(in case $d \neq 2$, of course, the same holds with $\sqrt{n}$ replaced by $n^{(d-1) / d}$ even if $\gamma(n)=n^{2}$ ). This type of convergence is called complete convergence (cf. [St (81b)]).

Although Theorem 2.1 seems to be much more general and stronger than Propositions 1.1 and 1.2, its proof is quite straightforward by using ideas from [St (81b)] and [Rh (86)]. In particular, our main tool will be the following inequality, due to Efron and Stein, which is also used in the two papers mentioned above:

LEMMA 2.3 (cf. [ES (78)]). Let $S\left(x_{1}, \ldots, x_{n-1}\right)$ be any symmetric function of $n-1$ variables $x_{i}$ and let $X_{i}$ be i.i.d. random variables. Furthermore, define new random variables by

$$
S_{i}^{n}=S\left(X_{1}, \ldots, X_{i-1}, X_{i+1}, \ldots, X_{n}\right) \quad(i=1, \ldots, n)
$$

and $\bar{S}=(1 / n) \sum_{i=1}^{n} S_{i}^{n}$.

Then the following inequality holds:

$$
\operatorname{Var} S\left(X_{1}, \ldots, X_{n-1}\right) \leqslant E \sum_{i=1}^{n}\left(S_{i}^{n}-\bar{S}\right)^{2} .
$$

Now let us turn to the proof of Theorem 2.1: Let $n \in \mathbb{N}$ be fixed. Replace $f_{i}^{n}$ by the more convenient notion $f_{i}$. Let $\phi$ be any smooth function. Then, by Lemma 2.3,

$$
\operatorname{Var} \phi\left(f_{n}\right) \leqslant E \sum_{i=1}^{n}\left(\phi\left(f_{i}\right)-\bar{\phi}\right)^{2}
$$

where $\bar{\phi}=(1 / n) \sum_{i=1}^{n} \phi\left(f_{i}\right)$.

Replacing $\phi$ by any other function of $X_{1}, \ldots, X_{n}$ only increases the right-hand side of (1), thus we get

$$
\operatorname{Var} \phi\left(f_{n}\right) \leqslant E \sum_{i=1}^{n}\left(\phi\left(f_{i}\right)-\phi\left(f_{k}\right)\right)^{2} \quad \forall k=1, \ldots, n .
$$

Summing up these inequalities for $k=1, \ldots, n$, we get

$$
\operatorname{Var} \phi\left(f_{n}\right) \leqslant \frac{1}{n} E \sum_{i=1}^{n} \sum_{k=1}^{n}\left(\phi\left(f_{i}\right)-\phi\left(f_{k}\right)\right)^{2}
$$

By the mean value theorem, we can choose a value $\beta_{i k}$ between $f_{i}$ and $f_{k}$ such that

$$
\phi\left(f_{i}\right)-\phi\left(f_{k}\right)=\phi^{\prime}\left(\beta_{i k}\right)\left(f_{i}-f_{k}\right) \text {. }
$$

Furthermore, let us assume that $\phi^{\prime}$ is nonnegative and increasing. Thus, (4) implies

$$
\begin{aligned}
\left|\phi\left(f_{i}\right)-\phi\left(f_{k}\right)\right| & \leqslant \phi^{\prime}\left(f_{i}+\left|f_{i}-f_{k}\right|\right) \cdot\left|f_{i}-f_{k}\right| \\
& \leqslant \phi^{\prime}\left(f_{n}+\left|f_{i}-f_{n}\right|+\left|f_{i}-f_{k}\right|\right)\left|f_{i}-f_{k}\right| .
\end{aligned}
$$

Using (3) and (5), we conclude that

$$
\operatorname{Var} \phi\left(f_{n}\right) \leqslant \frac{1}{n} E \sum_{i} \sum_{k}\left[\phi^{\prime}\left(f_{n}+\left|f_{i}-f_{n}\right|+\left|f_{i}-f_{k}\right|\right)\left|f_{i}-f_{k}\right|\right]^{2} .
$$


Now it seems promising to choose $\phi$ as an exponential function in order to make $\phi$ appear on both sides of (6). At this moment, however, let us specify $\phi$ only up to a parameter $\alpha>0$ to be determined later. Thus define

$$
\phi_{\alpha}(x):=e^{\alpha\left(x-E f_{n}\right) \sqrt{n / \gamma(n)}} .
$$

(6) then becomes

$$
\begin{aligned}
\operatorname{Var} \phi_{\alpha}\left(f_{n}\right) & \leqslant \frac{1}{n} E \sum_{i} \sum_{k} \alpha^{2} \frac{n}{\gamma(n)}\left[\phi_{\alpha}\left(f_{n}+\left|f_{i}-f_{n}\right|+\left|f_{i}-f_{k}\right|\right)\left|f_{i}-f_{k}\right|\right]^{2} \\
& \leqslant \frac{\alpha^{2}}{\gamma(n)} E \sum_{i} \sum_{k}\left[\phi_{\alpha}\left(f_{n}\right) \cdot e^{2 \alpha A \sqrt{n / \gamma(n)}}\left|f_{i}-f_{k}\right|\right]^{2}
\end{aligned}
$$

(the latter inequality being due to $\left|f_{i}-f_{k}\right| \leqslant A$ for all $i, k$ ).

By our assumption, $\gamma(n) \geqslant n$; thus

$$
e^{2 \alpha A \sqrt{n / \gamma(n)}} \leqslant 2 \text { for } \alpha \leqslant \frac{1}{4} A .
$$

Thus, assume $\alpha \leqslant \frac{1}{4} A$ in what follows. Then, using the bound on $\sum_{i} \sum_{k}\left|f_{i}-f_{k}\right|^{2}$, we conclude from (7) that

$$
\operatorname{Var} \phi_{\alpha}\left(f_{n}\right) \leqslant 4 B \alpha^{2} E\left[\phi_{\alpha}\left(f_{n}\right)^{2}\right]
$$

Writing $\phi_{\alpha}$ for short instead of $\phi_{\alpha}\left(f_{n}\right)$ and noting that $\left(\phi_{\alpha}\right)^{2}=\phi_{2 \alpha}$, the last inequality becomes

$$
\begin{aligned}
& E \phi_{2 \alpha}-\left(E \phi_{\alpha}\right)^{2}=\operatorname{Var} \phi_{\alpha} \leqslant 4 B \alpha^{2} E \phi_{2 \alpha} \text { or } \\
& E \phi_{\alpha}-\left(E \phi_{\alpha / 2}\right)^{2} \leqslant B \alpha^{2} E \phi_{\alpha} \text { or }
\end{aligned}
$$

$$
E \phi_{\alpha} \leqslant\left(1-B \alpha^{2}\right)^{-1}\left(E \phi_{\alpha / 2}\right)^{2}
$$

Now we specify $\alpha=\min \left(\frac{1}{4} A, \frac{1}{2} \sqrt{1 / B}\right)$ and proceed as in [Rh (86)]: By induction, (9) yields

$$
\begin{aligned}
E \phi_{\alpha} & \leqslant\left(1-B \alpha^{2}\right)^{-1}\left(1-B\left(\frac{\alpha}{2}\right)^{2}\right)^{-2}\left(E \phi_{\alpha / 4}\right)^{4} \\
& \vdots \\
& \leqslant \prod_{i=0}^{k-1}\left(1-B\left(\frac{\alpha}{2^{i}}\right)^{2}\right)^{-2^{i}}\left(E \phi_{\alpha / 2^{k}}\right)^{2^{k}}
\end{aligned}
$$

The infinite product $\prod_{i=0}^{\infty}\left(1-B\left(\alpha / 2^{i}\right)\right)^{-2^{i}}$ converges, since so does the series

$$
\sum_{i=0}^{\infty} 2^{i} B\left(\frac{\alpha}{2^{i}}\right)^{2}=B \sum_{i=0}^{\infty} \frac{\alpha}{2^{i}} .
$$

Furthermore, it follows from Lemma 3 of [Rh (86)] that $\lim _{k \rightarrow \infty}\left(E \phi_{\alpha / 2^{k}}\right)^{2^{k}}=1$. We include the short proof for completeness: Let $h(t)=E \phi_{\alpha t}$. Then $h$ is continuously 
differentiable with $h(0)=1$ and $h^{\prime}(0)=1$ and $h^{\prime}(0)=0$. Thus $h(t)=1+o(t)$ and hence $\lim _{t \rightarrow 0}[h(t)]^{1 / t}=1$.

Hence there exists a constant $\beta>0$ (depending only on $\alpha$, and hence on $A$ and $B$ ) such that

$$
\begin{gathered}
E \phi_{\alpha} \leqslant \prod_{i=0}^{\infty}\left(1-B\left(\frac{\alpha}{2^{i}}\right)^{2}\right)^{-2^{i}} \lim _{k \rightarrow \infty}\left(E \phi_{\alpha / 2^{k}}\right)^{2^{k}} \leqslant \frac{\beta}{2}, \\
\text { i.e. } E e^{\alpha\left(f_{n}-E f_{n}\right) \sqrt{n / \gamma(n)}} \leqslant \frac{\beta}{2} \text { or } E e^{\alpha\left|f_{n}-E f_{n}\right| \sqrt{n / \gamma(n)}} \leqslant \beta .
\end{gathered}
$$

Thus, for $t>0$,

$$
\begin{gathered}
\operatorname{Prob}\left(\alpha\left|f_{n}-E f_{n}\right| \sqrt{\frac{n}{\gamma(n)}} \geqslant t\right) e^{t} \leqslant E e^{\alpha\left|f_{n}-E f_{n}\right| \sqrt{n / \gamma(n)}} \leqslant \beta \\
\text { or } \operatorname{Prob}\left(\left|f_{n}-E f_{n}\right| \geqslant t\right) \leqslant \beta e^{-\alpha t \sqrt{n / \gamma(n)}}
\end{gathered}
$$

which proves the theorem.

REMARK. As pointed out by the referees, Wan Soo T. Rhee and M. Talagrand (cf. [RT (86a), (b)]) have recently discovered that martingale inequalities may be used to obtain stronger results for the two problems considered above (Bin packing and Shortest Path). In particular, their method yields that, for some constant $\alpha>0$,

$$
\operatorname{Prob}\left(\left|Y_{n}-E Y_{n}\right|>t\right) \leqslant 2 e^{-\alpha t^{2} / \log (1+t)}
$$

in case $Y_{n}$ denotes the Shortest Path through uniformly distributed independent random variables $X_{1}, \ldots, X_{n}$. However, we could not see, how to derive a general result like Theorem 2.1, using the technique of Rhee and Talagrand.

Acknowledgement. I am grateful to the referees and Professor E. Cinlar for many helpful comments. In particular, our original version of Lemma 2.2 has been improved by one of the referees.

\section{References}

[BHH (59)] Bearwood, J., Halton, J. H. and Hammersley, J. M. (1959). The Shortest Path through Many Points. Proc. Cambridge Philos. Soc. 55, 299-327.

[ES (78)] Efron, B. and Stein, C. (1978). The Jackknife Estimate of Variance. Technical Report No. 120, Dept. of Statistics, Stanford University.

[Ki (76)] Kingman, J. F. C. (1976). Subadditive Processes. Lecture Notes in Math. 539, Springer-Verlag, Berlin and New York, 168--222.

[Rh (86)] Rhee, Wan Soo T. (1986). Convergence of Optimal Stochastic Bin Packing. Working paper, Ohio State University.

[RT (86a)] _ and Talagrand, M. (1987). Martingale Inequalities and NP-Complete Problems. Math. Oper. Res. 12, 1 177-181.

[RT (86b)] _ (1986). Martingale Inequalities, Interpolation and NP-Complete Problems. Tech. Report, Ohio State University.

[St (81a)] Steele, J. M. (1981). Subadditive Euclidean Functionals and Nonlinear Growth in Geometric Probability. Ann. Probab. 9, 3 365-376.

[St (81b)] (1981). Complete Convergence of Short Paths and Karp's Algorithm for the TPS. Math. Oper. Res.

MATHEMATISCHES INSTITUT, UNIVERSITAT KOLN, WEYERTAL 86-90, D-5000 KOLN 41, FEDERAL REPUBLIC OF GERMANY

Current address: UNIVERSITY OF TWENTE, P.O. BOX 217, 7500 AE ENSCHEDE, THE NETHERLANDS 